\title{
Divergent Differentiation
}

National Cancer Institute

\section{Source}

National Cancer Institute. Divergent Differentiation. NCI Thesaurus. Code C104333.

A microscopic finding indicating that a tumor exhibits more than one line of histologic differentiation. 